\title{
Erratum: Towards first-principles prediction of valence instabilities in mixed stack charge-transfer crystals [Phys. Rev. B 95, 155125 (2017)]
}

\author{
Francesca Delchiaro, Alberto Girlando, Anna Painelli, Arkamita Bandyopadhyay, Swapan K. Pati, and Gabriele D'Avino
}

(Received 21 October 2018; published 5 November 2018)

DOI: 10.1103/PhysRevB.98.199901

In the original paper, we have discussed the valence instabilities of several mixed stack charge-transfer (CT) crystals following the approach proposed by D'Avino and Verstraete [1] that combines modified Hubbard model calculations with atomistic calculations to provide system-specific model parameters. In the present Erratum, we amend incorrect results in our paper concerning the calculation of the electrostatic (Madelung) energies and discuss their implications for the conclusions of our paper.

\section{CORRECT CALCULATION OF THE MADELUNG ENERGIES}

Following Ref. [1], in the original paper, the Madelung energy was computed as the difference between the electrostatic energy of the ionic $(I)$ and the neutral $(N)$ lattices,

$$
M=E(I)-E(N),
$$

where the electrostatic energies were evaluated with an atomic point-charge model,

$$
E(N / I)=\frac{1}{2 N_{m}} \sum_{m i} q_{m i}^{(N / I)} \phi_{m i}^{(N / I)}
$$

The sum above runs on the atoms in the unit cell, $q_{m i}^{(N / I)}$ and $\phi_{m i}^{(N / I)}$ are the charge and the electric potential at atom $i$ of molecule $m$ in the $N / I$ lattice. $N_{m}$ is the number of molecules in the unit cell. We remark that the present definition of $M$ differs by a factor of 2 from the standard one. In fact, Madelung energies are usually defined per ion pair [i.e., per donor-acceptor (DA) pair in our case], whereas Eq. (2) quantifies the intensive energy per molecule.

The evaluation of Madelung sums is notably a delicate issue due to the long-range nature of Coulomb interactions that leads to conditionally convergent sums [2-5]. In our paper and Ref. [1] we have opted for a direct summation approach that includes all the periodic replicas of the crystal unit cell within a spherical cutoff distance, which is chosen large enough to converge energies within 10-meV accuracy. The inclusion of entire unit cells ensures the charge neutrality of the spherical cluster over which the sum is performed.

We have now realized, and report here, that this approach may yield incorrect results due to the occurrence of surface charges resulting from the uncompensated dipoles of the (open boundary) unit cell. This issue is particularly relevant for ionic lattices where the cell dipole can be large due to the presence of charged molecules and/or for crystals with one DA unit per unit cell. In order to illustrate the problem, we consider first a hypothetical mixed stack CT crystal with one unit formula (DA pair) in the unit cell as, for instance, in 4, 4'-dimethyltetrathiafulvalene-chloranil (DMTTF-CA) at room temperature (see our paper). As shown in Fig. 1(a), the cell has a large dipole moment $e a, a$ being the lattice spacing and $e$ being the elementary charge. When the cell is replicated in three dimensions, the dipoles in the inner part of the sphere cancel out (we are dealing with regular, i.e., nondimerized stacks, which have zero polarization by the symmetry) but not those at the surface.

Surface charges are an artifact due to the use of a finitesystem approach to compute an infinite crystal. The effect of

(a)
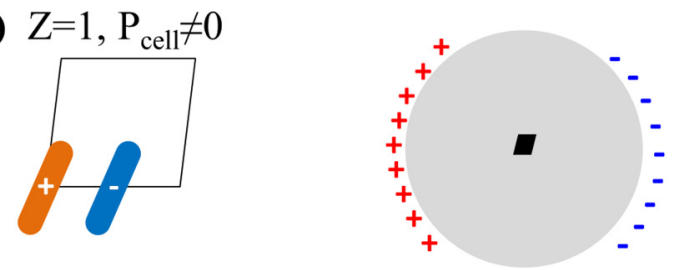

(b)
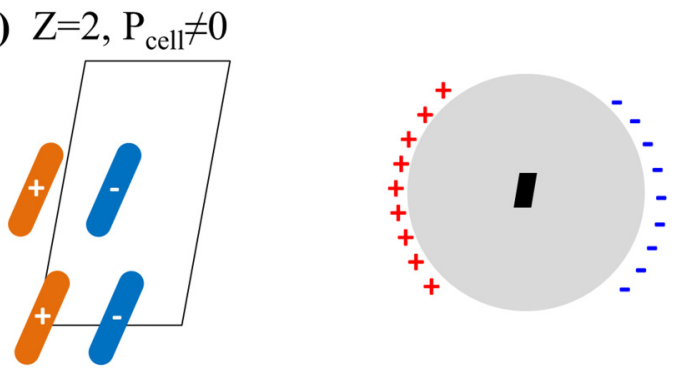

(c)
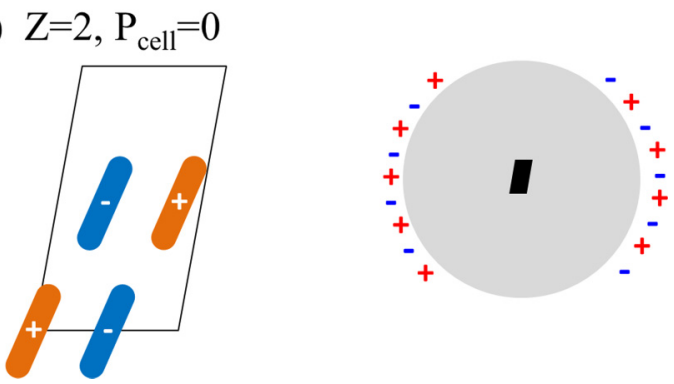

FIG. 1. Illustration of the effect of surface charges in the evaluation of electrostatic energies in mixed stack CT crystals with (a) one and (b) and (c) two collinear DA pairs in the unit cell. Note the difference in surface charges for different, albeit equivalent, choices of molecular position in the unit cells in panels (b) and (c). 
TABLE I. Model parameters for the modified Hubbard model with the corrected value for $M$ and $\epsilon_{c}$. All parameters have eV energy units.

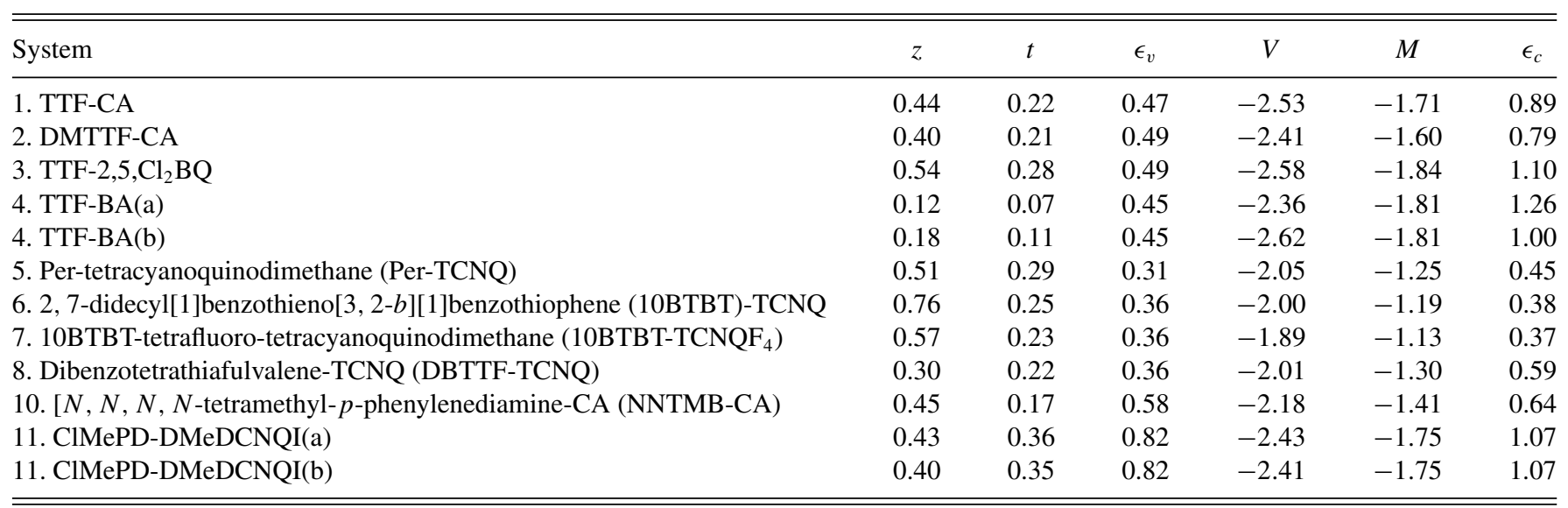

surface charges can be eliminated using classical electrostatics results for the field inside a uniformly polarized sphere [6]. The potential in Eq. (2) should then be replaced by (CGS units)

$$
\tilde{\phi}(\mathbf{r})=\phi(\mathbf{r})-\frac{4 \pi}{3} \mathbf{P} \cdot \mathbf{r},
$$

where $\mathbf{P}$ is the unit-cell (open boundary) dipole moment per unit volume. We underline that $\mathbf{P}$ as defined in the paper does not measure the crystal polarizability, that actually vanishes in all considered structures. Finite-P values are obtained due to surface charges in the large but finite portion of the crystal we use for Madelung calculations.

We now consider the case of a mixed stack crystal with two DA pairs in the unit cell, leading to two parallel stacks, as in tetrathiafulvalene-chloranil (TTF-CA, see the original paper and Ref. [1]). In this case one can choose a unit cell where the dipoles of the two stacks sum up [see Fig. 1(b)] or cancel out [see Fig. 1(c)]. The first case is similar to the one with one DA pair in the unit cell. The electrostatic energy must be, however, independent of the choice of equivalent unit cells. This has been explicitly numerically checked for the systems with two DA pairs per unit cell, demonstrating that the correction for surface charges in Eq. (3) do indeed compensate for the different dipoles of the unit cell.

We have then replicated all the relevant calculations, and the present Table I substitutes the corresponding Table II of the original paper. With the exception of system No. 10 (NNTMB-CA), the only modified values are $M$ and $\epsilon_{c}=V-$ $2 M$. In the case of NNTMB-CA the reported wrong values for the other parameters $\left(z, t, \epsilon_{v}\right.$, and $\left.V\right)$ due to a trivial transcription error. The Madelung energy for an ionic DA stack is clearly negative (attractive intermolecular interactions are expected) and approximately scales with the cell volume per DA pair. The variation of $\epsilon_{c}$ values in Table I is less important than previously stated, and no zero or negative values are found. Therefore the previous statement (see the original paper) that zero or negative $\epsilon_{c}$ values are indicative of important repulsive interstack interactions is incorrect.

The correction for surface charges is negligible in cases where the unit cells have zero dipoles as schematically shown in Fig. 1(c). An appropriate choice of the unit-cell geometry therefore ensures correct results for systems with two molecules per unit cell. This was the case for Ref. [1] and for TTF-CA in our paper, although the result in Table I is more accurate with all shown decimal digits converged. Larger discrepancies with respect to the original paper are instead found for systems with one DA pair in the unit cell and for the ionic tetrathiafulvalene-bromanil (TTF-BA) in which the two stacks are almost orthogonal and the corresponding dipoles sum up.

\section{IMPLICATIONS OF THE MADELUNG ENERGY CORRECTION}

We now analyze the consequences of the new values of the Madelung energy on the prediction of the ground-state properties of mixed stack CT crystals (Sec. IV of our paper). The present Fig. 2 replaces the corresponding one of our paper. From the universal $\rho\left(z_{\text {eff }} / t\right)$ curve (dashed black lines in all
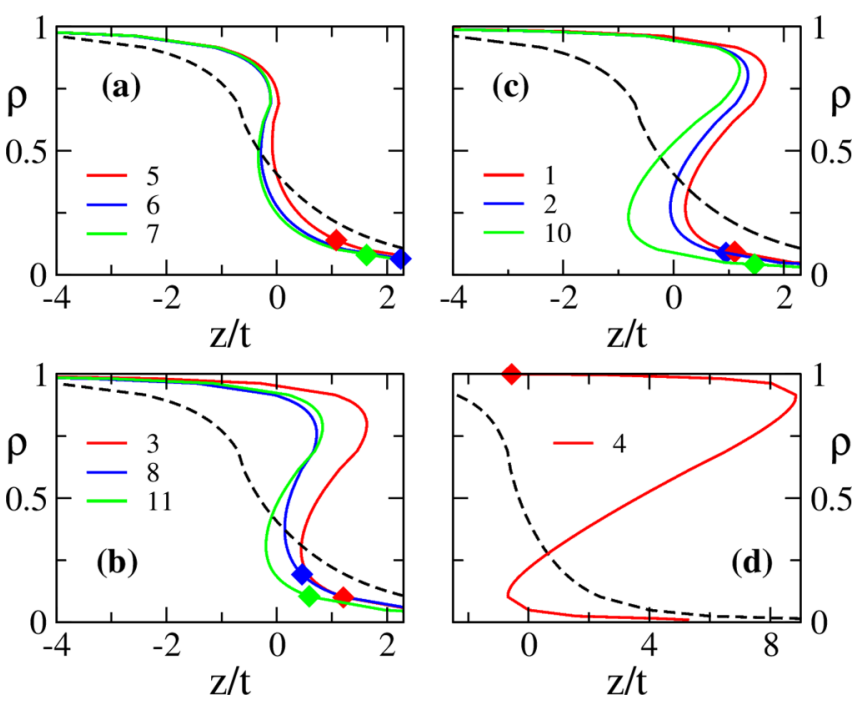

FIG. 2. Correct results of the modified Hubbard model calculations for the CT crystals. In all panels, the dashed black line is the universal $\rho(z / t)$ curve, and the colored lines refer to the specific systems numbered in the text. The diamonds show the ionicity computed for the $z^{\star} / t$ value obtained from the atomistic parametrization. 
TABLE II. Estimated model parameters entering the mean-field solution of the modified Hubbard model.

\begin{tabular}{lrrr}
\hline \hline System & $\epsilon_{T} / t$ & $\epsilon_{v} / t$ & $z^{*} / t$ \\
\hline 1. TTF-CA & 6.18 & 2.13 & 1.11 \\
2. DMTTF-CA & 6.10 & 2.38 & 0.95 \\
3. TTF-2, 5Cl 2 BQ & 5.68 & 1.76 & 1.21 \\
4. TTF-BA & 17.56 & 4.86 & -0.56 \\
5. Per-TCNQ & 2.62 & 1.07 & 1.08 \\
6. 10BTBT-TCNQ & 2.96 & 1.45 & 2.25 \\
7. 10BTBT-TCNQF & 3.17 & 1.60 & 1.63 \\
8. DBTTF-TCNQ & 4.32 & 1.61 & 0.46 \\
10. NNTMB-CA & 7.18 & 3.41 & 1.47 \\
11. CIMePD-DMeDCNQI & 5.35 & 2.30 & 0.59 \\
\hline \hline
\end{tabular}

panels of Fig. 2), we can extract the $\rho(z / t)$ curve specific for each investigated system using $\epsilon_{v} / t$ and $\epsilon_{T} / t=\left(\epsilon_{c}+\epsilon_{v}\right) / t$ listed in Table II. Finally, the estimated $z^{*} / t$ values in Table II, which updates the corresponding table of the original paper, fix the relevant abscissa point for each system at hand, then allowing for extracting a theoretical estimate for $\rho$, shown as diamonds in Fig. 2.

Figure 3 compares the correct calculated $\rho$ values with corresponding experimental estimates of room-temperature ionicity for the crystals (references in the figure caption). To make the comparison meaningful we set an error bar on the order of 0.15 on experimental estimates of $\rho$. The uncertainty on the calculated $\rho$ values is estimated as discussed in our paper. Results in Fig. 3 suggest that the described approach gives sufficiently reliable estimates of the ionicity, even if a general tendency to underestimate $\rho$ is observed.

We now comment on results in Fig. 2 where the ten considered systems are grouped based on the $\epsilon_{T} / t$ values. All calculated $\epsilon_{T} / t$ values are larger than the critical 1.8 value (see our paper) so that all calculated curves show bistable behavior. Three systems, however, Per-TCNQ, 10BTBTTCNQ, and 10BTBT-TCNQF 4 [Fig. 2(a)] have small $\epsilon_{T} / t$ 's, and the relevant curves are marginally bistable. Three systems, TTF-2, 5, $\mathrm{Cl}_{2} \mathrm{BQ}$, DBTTF-TCNQ, and CIMePDDMeDCNQI [Fig. 2(b)] with larger $\epsilon_{T} / t$ 's, show a moderate bistability. The remaining four systems, DMTTF-CA, CIMePD-DMeTCNQI, TTF-CA [Fig. 2(c)], and TTF-BA [Fig. 2(d)] show bistability with an increasingly sizable unaccessible region of intermediate $\rho$ values.

Comparing with experiment, we note that Per-TCNQ, 10BTBT-TCNQ, and 10BTBT-TCNQF 4 have small ionicities at room temperature as a consequence of the large energy gap $2 z$ (Table I) and are not prone to valence instability. For

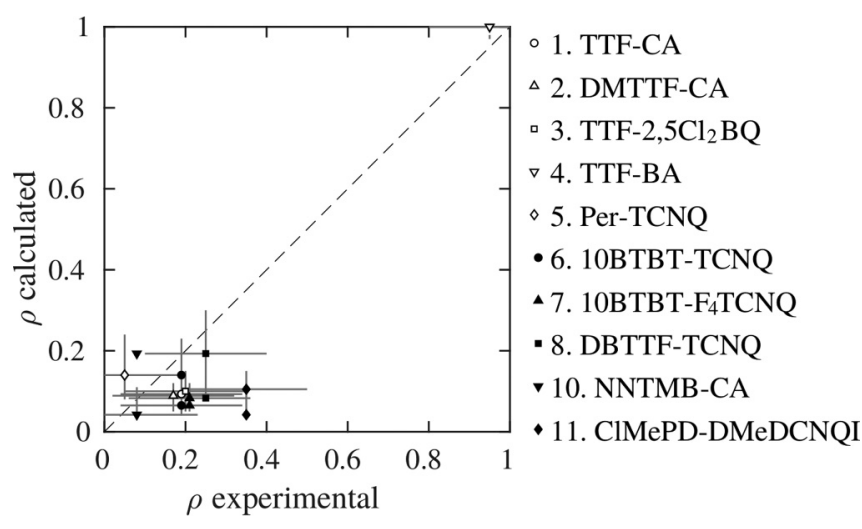

FIG. 3. Experimental $\rho$ values vs the corrected calculated values for the ten CT crystals considered in our paper.

TTF-CA [Fig. 2(c)] we predict that ionicities in the range from approximately 0.3 to 0.8 are not accessible, and indeed at room temperature, TTF-CA shows a regular stack structure with $\rho \sim 0.2$. Upon decreasing the temperature the ionicity of TTF-CA increases up to 0.3 and then, when reaching $81 \mathrm{~K}$, a discontinuous ionic phase transition occurs driving the system in the ionic regime with $\rho \sim 0.52$ and a dimerized stack structure (by further lowering the temperature, $\rho$ increases up to 0.6 at $10 \mathrm{~K}$ ) [7]. Experimentally, DMTTF-CA shows a much smaller charge instability region, whereas we predict a similar instability range as TTF-CA. Indeed, at $65 \mathrm{~K}$, DMTTF-CA undergoes a dimerization transition with $\rho$ increasing continuously or almost so from $\sim 0.2$ at room temperature to $\sim 0.4$ at $10 \mathrm{~K}$ [8]. TTF-2, $5 \mathrm{Cl}_{2} \mathrm{BQ}$ and DBTTF-TCNQ have room-temperature ionicity (between 0.2 and 0.3 ) similar to TTF-CA, yet they do not show any charge instability down to $10 \mathrm{~K}$. Indeed, we calculate for these systems smaller $\epsilon_{T}$ values than for TTF-CA (Table I), supporting a smaller tendency to instability. ClMePD-DMeDCNQI [Fig. 2(b)] is affected by disorder, which prevents long-range ordering: CIMePDDMeDCNQI presents a parallel increase in dimerization and ionicity, being the only example of a continuous ionicity change from quasineutral at room temperature $(\rho \approx 0.35)$ to quasi-ionic $(\rho \approx 0.6)$ at $80 \mathrm{~K}[9]$. Finally, for TTF-BA in Fig. 2(d) we predict an enormous instability region, which can be ascribed to the very small $t$ value and to the corresponding very large $\epsilon_{T} / t$ values. According to Fig. 2(d), TTF-BA can only be largely neutral with $\rho<0.15$ or largely ionic with $\rho>0.9$, and all intermediate ionicity values being forbidden. The estimated value of $z^{*} / t$ places the crystal on the ionic side, and indeed experimentally TTF-BA is largely ionic with $\rho>0.95$ and undergoes a dimerization transition upon decreasing temperature [10].
[1] G. D’Avino and M. J. Verstraete, Phys. Rev. Lett. 113, 237602 (2014).

[2] W. A. Harrison, Phys. Rev. B 73, 212103 (2006).

[3] A. Gellé and M.-B. Lepetit, J. Chem. Phys. 128, 244716 (2008).

[4] M. Gaio and P. L. Silvestrelli, Phys. Rev. B 79, 012102 (2009).
[5] E. I. Izgorodina, U. L. Bernard, P. M. Dean, J. M. Pringle, and D. R. MacFarlane, Crystal Growth and Design 9, 4834 (2009).

[6] C. Kittel, Introduction to Solid State Physics, 6th ed. (Wiley, New York, 1986). 
[7] M. Masino, A. Girlando, A. Brillante, R. G. D. Valle, and E. Venuti, Mater. Sci.-Pol. 22, 333 (2004).

[8] P. Ranzieri, M. Masino, A. Girlando, and M.-H. LeméeCailleau, Phys. Rev. B 76, 134115 (2007).
[9] S. A. Bewick, R. A. Pascal, D. M. Ho, Z. G. Soos, M. Masino, and A. Girlando, J. Chem. Phys. 122, 024710 (2005).

[10] A. Girlando, C. Pecile, and J. Torrance, Solid State Commun. 54, 753 (1985). 